\title{
Structure of Large-Amplitude Internal Solitary Waves
}

\author{
VASILIY VLASENKO* \\ Marine Hydrophysical Institute, Sevastopol, Ukraine \\ Peter Brandt† And Angelo Rubino\# \\ Institut für Meereskunde, Universität Hamburg, Hamburg, Germany
}

(Manuscript received 30 November 1998, in final form 7 October 1999)

\begin{abstract}
The horizontal and vertical structure of large-amplitude internal solitary waves propagating in stratified waters on a continental shelf is investigated by analyzing the results of numerical simulations and in situ measurements. Numerical simulations aimed at obtaining stationary, solitary wave solutions of different amplitudes were carried out using a nonstationary model based on the incompressible two-dimensional Euler equations in the frame of the Boussinesq approximation. The numerical solutions, which refer to different density stratifications typical for midlatitude continental shelves, were obtained by letting an initial disturbance evolve according to the numerical model. Several intriguing characteristics of the structure of the simulated large-amplitude internal solitary waves like, for example, wavelength-amplitude and phase speed-amplitude relationship as well as form of the locus of zero horizontal velocity emerge, consistent with those obtained previously using stationary Euler models. The authors' approach, which tends to exclude unstable oceanic internal solitary waves as they are filtered out during the evolution process, was also employed to perform a detailed comparison between model results and characteristics of large-amplitude internal solitary waves found in high-resolution in situ data acquired north and south of the Strait of Messina, in the Mediterranean Sea. From this comparison the importance of using higher-order theoretical models for a detailed description of large-amplitude internal solitary waves observed in the real ocean emerge. Implications of the results showing the complexity related to a possible inversion of sea surface manifestations of oceanic internal solitary waves into characteristics of the interior ocean dynamics are finally discussed.
\end{abstract}

\section{Introduction}

Internal solitary waves are thought to be ubiquitous in the World Ocean (Ostrovsky and Stepanyants 1989). In several places, for example, in the Andaman Sea (Osborne and Burch 1980), in the Sulu Sea (Apel et al. 1985), and at the Mascarene Ridge (Konyaev et al. 1995), large-amplitude internal solitary waves are observed, that is, their amplitudes and typical length scale of the vertical stratification are of the same order. Although they can evolve from a large amount of distur-

\footnotetext{
* Current affiliation: Institut für Mechanik, Technische Universität Darmstadt, Darmstadt, Germany.

$\dagger$ Current affiliation: Institut für Meereskunde, Universität Kiel, Kiel, Germany.

\# Current affiliation: Dipartimento di Dipartimento di Scienze Ambientali, Università di Venezia, Venice, Italy.
}

Corresponding author address: Peter Brandt, Institut für Meereskunde, Universität Hamburg, Troplowitzstr. 7, D-22529 Hamburg, Germany.

E-mail: brandt@ifm.uni-hamburg.de bances in the oceanic density field like those caused by riverine or glacial intrusions into coastal waters, oscillations of oceanic fronts, or abrupt atmospheric pressure or wind variations, internal solitary waves mainly arise from the disintegration of the internal tides generated by the interaction of a barotropic tidal flow with topographic features like, for example, strait sills, continental slopes, or sandbanks. Oceanic internal solitary waves are thus thought to exert a significant contribution to the dissipation of the rotational energy of the earthmoon system and to the mixing of different water masses in the ocean (Apel et al. 1995; Munk and Wunsch 1998). As internal solitary waves can be linked to different phenomena occurring within the water column like, for example, variations in water velocity, salinity, temperature, or in passive tracer concentration, or at the sea surface like, for example, modulation of the surface wave spectrum, surface wave breaking, or accumulation of surfactants, different measurement techniques can be employed for their detection. Among them, remote sensing has proved to be a powerful tool for studying oceanic internal solitary waves (Apel et al. 1975; Alpers et al. 1996) and for assessing their relevance to the recog- 
nition of larger-scale oceanic fluctuations: Brandt et al. (1999), for instance, demonstrated the possibility of inferring characteristics of the subbasin circulation in the central Mediterranean Sea by analyzing synthetic aperture radar images of internal solitary waves generated in the Strait of Messina.

The huge amount of observations of oceanic internal solitary waves made in the last decades finds its counterpart in a great number of theoretical studies and laboratory experiments devoted to understanding the general nature of this oceanic phenomenon. The simplest theoretical model capable of describing the dynamics of oceanic internal solitary waves in shallow waters is the Korteweg-de Vries (KdV) equation (see, e.g., Benney 1966). In this model nonlinear waves of invariant form can emerge from an initial disturbance as a result of the equilibrium between phase and amplitude dispersion. However, there are several limitations to the applicability of the KdV model to the dynamics of internal solitary waves in the real ocean: for instance, the capability of the $\mathrm{KdV}$ model of reproducing experimental results decreases as wave amplitudes increase. The acknowledgment of this limitation of the KdV model led to the development of different models containing higher-order nonlinearities. Among them are two-layer models as well as continuously stratified models. To the first category of extended models belong, for example, the equations proposed by Djordjevic and Redekopp (1978), Miles (1979, 1981), Koop and Butler (1981), Funakoshi and Oikawa (1986), and Miyata (1988). Note that in the last equation, which has been successfully used to explain laboratory observations of large-amplitude internal solitary waves (Michallet and Barthelemy 1998), the full nonlinearity of the shallow-water theory up to the first-order phase dispersion is included, that is, no limitation is imposed on the wave amplitude. To the second category belong, for example, the equations proposed by Lee and Beardsley (1974), Benney and Ko (1978), Gear and Grimshaw (1983), Vlasenko (1994), and Grimshaw et al. (1997). Note that all the aforementioned models represent either weakly nonlinear and/or weakly nonhydrostatic approximations of the equations of motion. In order to overcome this limitation in the description of large-amplitude internal solitary waves, numerical integration of the complete set of the fully nonlinear, nonhydrostatic Euler equations was also performed. In this regard two different approaches were attempted: 1) the integration of the stationary Dubreil-Jacotin-Long equation (Dubreil-Jacotin 1937; Long 1953; Davis and Acrivos 1967; Tung et al. 1982; Turkington et al. 1991; Brown and Christie 1998) and 2) the integration of the nonstationary Euler equations (Lamb and Yan 1996; Terez and Knio 1998). Using the first approach a very broad set of solitary wave solutions can be found, including waves characterized by a zone of closed circulation where areas of unstable stratified fluid may exist (Brown and Christie 1998). The second approach, which consists of producing internal solitary waves as a result of the evolution of an initial disturbance, tends to filter out unstable waves that, nevertheless, can be solutions of the stationary problem. Thus, although both approaches can be used to study characteristics of largeamplitude internal solitary waves, the first one emphasizes the mathematical complexity of nonlinear dispersive waves, while from the second one information inherent in their realistic manifestations in the ocean and/ or in the atmosphere can be more easily inferred.

In the present paper we study the structure of largeamplitude internal solitary waves by analyzing results of a nonstationary model based on the incompressible two-dimensional Euler equations and by analyzing highresolution in situ data acquired north and south of the Strait of Messina, in the Mediterranean Sea. The paper is organized as follows. In section 2 the numerical model is described. The analysis of numerical results revealing several intriguing characteristics of large-amplitude internal solitary waves and their observational evidence are presented in section 3. Finally, in section 4, the results are discussed and conclusions are presented.

\section{The model}

\section{a. Model theory}

The numerical model used in the present investigation is based on the incompressible two-dimensional Euler equations in the frame of the Boussinesq approximation. The model is capable of describing the dynamics of a continuously stratified fluid in a vertical plane. Note that, in this model, the Coriolis force is neglected and the water depth $H$ is constant. In the following we refer the model equations to a Cartesian $x-z$ coordinate system with the $z$ axis directed vertically upward. In this frame, the equations that constitute our model are

$$
\begin{aligned}
& \Omega_{t}+J(\Omega, \Psi)=g \frac{\rho_{x}}{\rho_{a}}, \\
& \rho_{t}+J(\rho, \Psi)+\frac{\rho_{a}}{g} N^{2} \Psi_{x}=0 .
\end{aligned}
$$

Here $\Omega(x, z, t)$ is the vorticity,

$$
\Omega=\Psi_{x x}+\Psi_{z z},
$$

and $\Psi(x, z, t)$ represents the two-dimensional streamfunction,

$$
\Psi_{z}=u, \quad \Psi_{x}=-w,
$$

where $u(x, z, t)$ and $w(x, z, t)$ are the horizontal and vertical velocity components in the $x$ and $z$ direction respectively. The reference density (which in our model is the sea surface density), the density anomaly, and the undisturbed density profile are denoted by $\rho_{a}, \rho(x, z, t)$, and $\rho_{0}(z)$, respectively; $N(z)=\left(-g\left(\rho_{0}\right)_{z} / \rho_{a}\right)^{1 / 2}$ is the undisturbed Brunt-Väisälä frequency, and $J$ the Jacobian operator: $J(A, B)=A_{x} B_{z}-A_{z} B_{x}$. At the sea surface 
$(z=0)$ and at the bottom $(z=-H)$ the following boundary conditions are imposed:

$$
\Psi=0, \quad \Omega=0, \quad \rho=0 .
$$

For the simulation of the dynamics of internal solitary waves, which will be presented in the following section, the numerical model is integrated over a domain moving with the phase velocity $c_{f}$ of the leading wave (it corresponds to the fastest disturbance propagating in the positive $x$ direction, i.e., to the right). This implies that the disturbances generated during the adjustment phase move to the left of the model domain and never reach the leading wave again, as their phase velocities must be smaller than $c_{f}$. The right-hand boundary $x=x_{r}$ as well as the left-hand boundary $x=x_{l}$ are located far from the origin $x=0$, which coincides with the position of the maximum isopycnal displacement of the leading wave. The following boundary conditions are imposed at $x=x_{r}$ :

$$
\Psi=0, \quad \Omega=0, \quad \rho=0
$$

and at $x=x_{l}$ :

$$
\frac{\partial \Psi}{\partial x}=0, \quad \frac{\partial \Omega}{\partial x}=0, \quad \frac{\partial \rho}{\partial x}=0 .
$$

The boundary conditions (6) correspond to an assumed undisturbed ocean for $x \geq x_{r}$. As noted before, all disturbances traveling with a phase velocity smaller than $c_{f}$ will, sooner or later, leave the model domain at $x=$ $x_{l}$. Thus, in principle, the model results are independent of the particular choice of the boundary conditions there. Therefore, these boundary conditions are chosen solely to ensure numerical stability, as they avoid the growth of strong gradients in the model region near $x=x_{l}$, which could arise from numerical inaccuracies. Equations (1)-(2) with the boundary conditions (5)-(7) are solved numerically by using the splitting-up scheme discussed in detail by Marchuk (1974): The vorticity transport equation is integrated in time using the secondorder Crank-Nicholson scheme, while the spatial derivatives are approximated using a second-order central difference scheme. At each temporal step an implicit system of equations in the form of a tridiagonal matrix is obtained that is solved using standard techniques. The streamfunction is then computed from the vorticity using the Laplace equation (3). For its solution a standard relaxation method is used. Finally the density $\rho$ is computed using the same methodology used for the vorticity. The temporal spacing $\Delta t$ was selected to satisfy the Courant-Friedrichs-Levy condition: $\Delta x / \Delta t>c_{f}$, where $\Delta x$ represents the spatial grid step along the $x$ axis. The calculations were carried out using spatial grid steps $\Delta x$ $=2 \mathrm{~m}$ and $\Delta z=1 \mathrm{~m}$. The lattice on which the calculations were performed consists of 4000 nodes in the $x$ direction. In the $z$ direction the amount of nodes depends on the water depth.

\section{b. Model setup}

The model was initialized using analytical solitary waves solutions of the stratified $\mathrm{KdV}$ equation (see, e.g., Vlasenko 1994):

$$
\Psi(x, z, t)=-A V \operatorname{sech}^{2}\left(\frac{x-V t}{L}\right) W_{n}(z) .
$$

Here $A$ represents the wave amplitude (i.e., the maximum isopycnal displacement of the solitary wave), $V$ its phase speed

$$
V=\frac{N_{\max } H}{\sqrt{\mu_{n}}}\left(1-\frac{A \gamma}{3 \delta H \mu_{n}}\right),
$$

and $L$ its horizontal length scale

$$
L=\sqrt{-\frac{6 H^{3}}{A \gamma}} .
$$

Here $N_{\max }$ is the maximum Brunt-Väisälä frequency,

$$
\delta=\frac{\int_{-H}^{0} W_{n}^{2} N^{2} d z}{\int_{-H}^{0} W_{n}^{2} d z},
$$

and

$$
\gamma=-\mu_{n} \frac{\int_{-H}^{0} W_{n}^{3}\left(N^{2}\right)_{z} d z}{\int_{-H}^{0} W_{n}^{2} d z} .
$$

The parameters $\mu_{n}$ and $W_{n}(z)$ are the eigenvalues and the eigenfunctions of the boundary value problem:

$$
\begin{gathered}
\left(W_{n}\right)_{z z}+\mu_{n} N^{2} W_{n}=0 \\
\text { with } W_{n}(0)=W_{n}(-H)=0,
\end{gathered}
$$

where $n$ is the mode number. To initialize our numerical model, we used first-mode $(n=1)$ internal solitary wave solutions of the stratified $\mathrm{KdV}$ equation with different amplitudes. Inserting (8) into (3), the vorticity associated to the analytical internal solitary waves was obtained. Imposing the condition of isopycnic motion $\rho$ $=\rho(\Psi-V z)$, the density anomaly was calculated from the undisturbed density profile. The initial fields described above represent stationary solitary wave solutions in a weakly nonlinear, weakly nonhydrostatic medium. Once inserted into a nonstationary Euler model, they will evolve toward new stationary solitary wave solutions. The model is thus run until stationary solutions are reached. Thus using this method internal solitary waves of different amplitudes can be generated. In order to control that stationary solitary waves were indeed produced the following strategy was applied: As the change in shape, amplitude, velocities, and energy was less than $0.1 \%$ over a period of $20 T$, where 

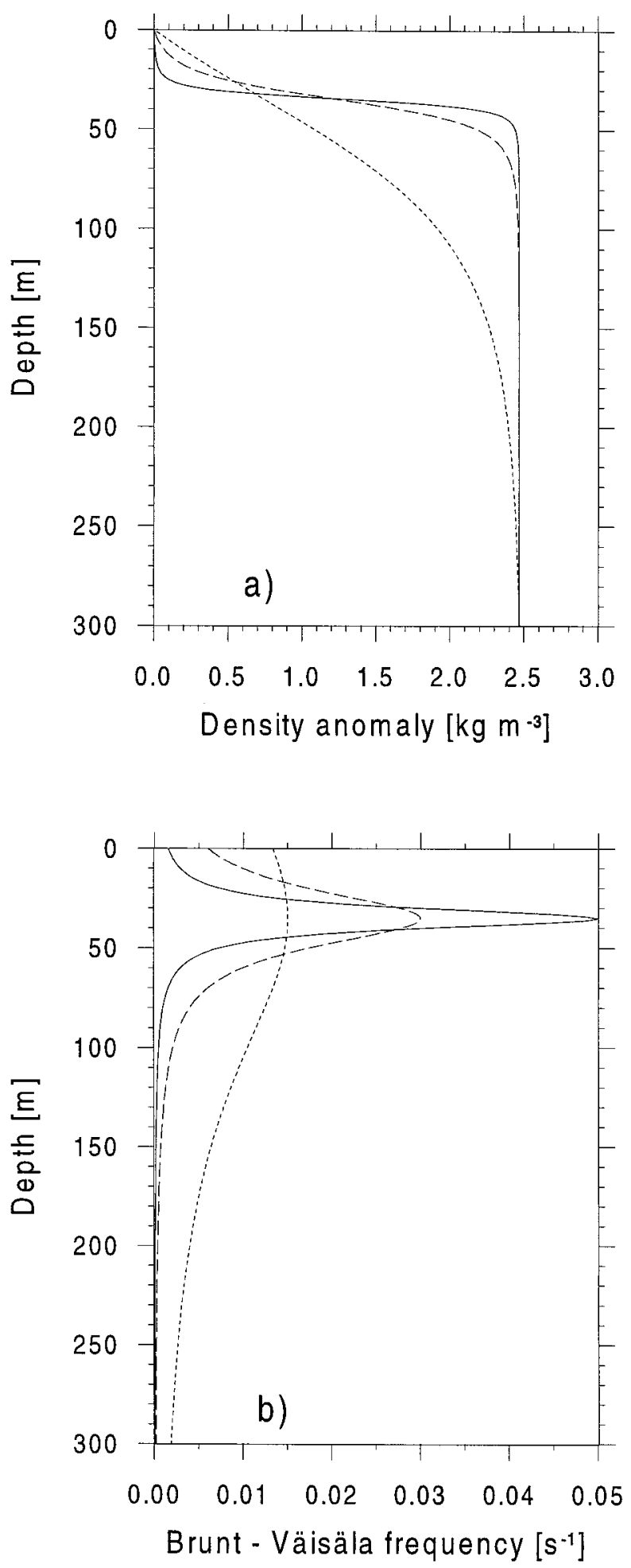

FIG. 1. Vertical profiles of (a) density anomalies and (b) BruntVäisälä frequencies characterizing the three density stratifications (see Table 1) for which numerical simulations were carried out using our Euler model. Solid lines refer to stratification 1, dashed lines to stratification 2, and dotted lines to stratification 3. The Brunt-Väisälä frequencies are computed using Eq. (14) with the three sets of parameters listed in Table 1.
TABLE 1. Values of the parameters characterizing the three water stratifications [see Eq. (14)] used in the numerical simulations.

\begin{tabular}{clcc}
\hline \hline $\begin{array}{c}\text { Stratifi- } \\
\text { cation }\end{array}$ & $\begin{array}{c}N_{\max } \\
\left(\mathrm{s}^{-1}\right)\end{array}$ & $\begin{array}{c}H_{N} \\
(\mathrm{~m})\end{array}$ & $\begin{array}{c}L_{N} \\
(\mathrm{~m})\end{array}$ \\
\hline 1 & 0.05 & 35 & 12.35 \\
2 & 0.03 & 35 & 35 \\
3 & 0.015 & 35 & 196.5 \\
\hline
\end{tabular}

$T=L / c_{f}$, the wave was considered as a stationary solitary wave.

\section{Characteristics of large-amplitude internal solitary waves}

\section{a. Model results}

In this section we discuss several characteristics of large-amplitude internal solitary waves inferred from our nonstationary Euler model for three different density stratifications as illustrated in Fig. 1. The Brunt-Väisälä frequencies characterizing the three stratifications belong to the following three-parameter family of curves:

$$
N(z)=N_{\max }\left(\left[\frac{2\left(z+H_{N}\right)}{L_{N}}\right]^{2}+1\right)^{-1} .
$$

Here $H_{N}$ represents the depth where the Brunt-Väisälä frequency is maximum and $L_{N}$ a vertical length scale characterizing the Brunt-Väisälä frequency variation. In Table 1 the values of $N_{\max }, H_{N}$, and $L_{N}$ used for determining the three stratifications are listed. These stratifications represent typical water mass distributions on midlatitude continental shelves (in the simulations the water depth is assumed to be $300 \mathrm{~m}$ ) characterized by the presence of a seasonal pycnocline of different strength. Note that the three different density profiles share the same surface and bottom density values.

The following discussion is devoted to elucidate different characteristics of large-amplitude internal solitary waves, which, as a result of our numerical simulations, emerge as general peculiarities of these oceanic features. For the sake of clarity we will thus present here only results obtained using the stratification identified by the second set of parameters given in Table 1.

Figure $2 \mathrm{a}$ shows the density field of a large-amplitude internal solitary wave as obtained by using the numerical Euler model (solid lines) and as calculated by using the analytical KdV model (dashed lines) for the same density stratification. Both waves have the same amplitude, that is, their maximum isopycnal displacements coincide. In a near-surface layer of about $40 \mathrm{~m}$ thickness the isopycnal displacements of the Euler internal solitary wave exceed those of the KdV internal solitary wave. In the lower layer the opposite occurs. A remarkable difference between Euler and KdV internal solitary waves can be noted by comparing their wavelengths. In the present case the wavelength of the Euler 


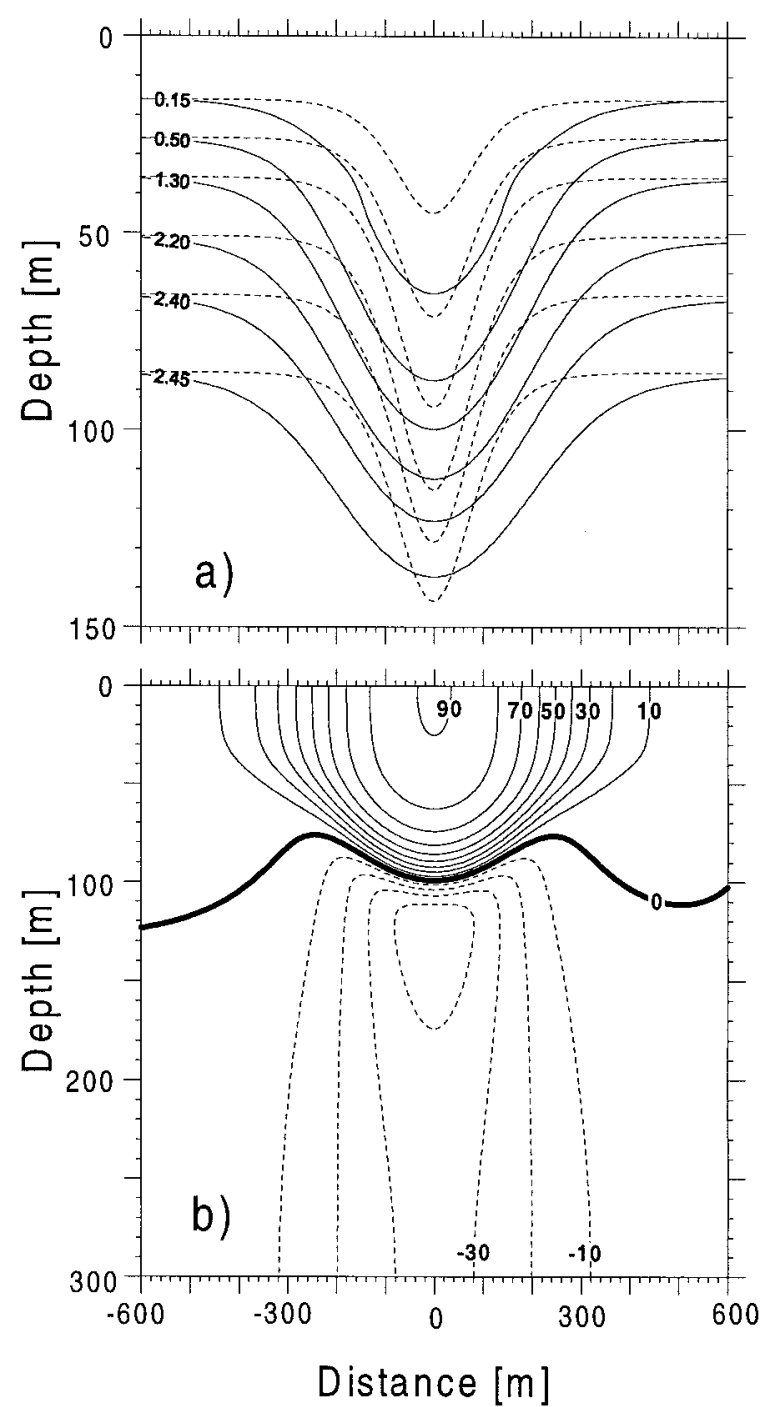

FIG. 2. Fields of (a) density anomaly $\left(\mathrm{kg} \mathrm{m}^{-3}\right)$ and (b) horizontal velocity $\left(\mathrm{cm} \mathrm{s}^{-1}\right)$ of a large-amplitude internal solitary wave obtained using stratification 2 (see Table 1). In (a) the solid lines refer to the results of our Euler model, the dotted lines to the results of the KdV model. Panel (b) refers uniquely to the results of our Euler model Solid lines denote positive horizontal velocities and dotted lines negative horizontal velocities.

internal solitary wave is larger than that of the $\mathrm{KdV}$ internal solitary wave. The dependence of the wavelength of Euler internal solitary waves on density stratification and wave amplitude is, however, nontrivial. It will be discussed later. Figure $2 \mathrm{~b}$ shows the horizontal velocity field of the same Euler internal solitary wave depicted in Fig. 2a. The form of the locus of zero horizontal velocity (heavy solid line) differs remarkably from the horizontal straight line that would result from the $\mathrm{KdV}$ model. Note that the minimum horizontal velocity is not located at the bottom, like in the $\mathrm{KdV}$ solution. This indicates that the modal structure of Euler internal solitary waves is affected by the higher-order nonlinearity.
Figure 3 shows the vertical structure of Euler and $\mathrm{KdV}$ internal solitary waves of different amplitudes for the same density stratification discussed above. Depicted are vertical profiles of (a) the normalized isopycnal displacements calculated at the wave center, (b) the normalized horizontal velocities calculated at the wave center, and (c) the normalized vertical velocities calculated at the positions of maximum vertical velocity. The following parameters of $\mathrm{KdV}$ internal solitary waves having the same amplitude as the presented Euler internal solitary waves were used for the normalization: (a) wave amplitude $\left(A_{\mathrm{KdV}}\right)$, (b) maximum horizontal velocity $\left(U_{\mathrm{KdV}}\right)$, and (c) maximum vertical velocity $\left(W_{\mathrm{KdV}}\right)$. Choosing this normalization, the resulting vertical profiles of the $\mathrm{KdV}$ internal solitary waves are independent on wave amplitude. Euler internal solitary waves possess several characteristics that differ from KdV internal solitary waves. In fact, depth of maximum isopycnal displacement (see Fig. 3a), depth of zero horizontal velocity (see Fig. 3b), and depth of maximum vertical velocity (see Fig. 3c) are functions of wave amplitude: While the first parameter decreases, the second and third increase with increasing wave amplitude. In general, the difference between Euler and $\mathrm{KdV}$ internal solitary waves increases with increasing amplitude. Note that the maximum horizontal velocity as well as the vertical velocities of Euler internal solitary waves are smaller than those of the $\mathrm{KdV}$ internal solitary waves, independent of wave amplitude. This is a consequence of the fact that, for this stratification, in the $\mathrm{KdV}$ model nonlinear effects are overestimated.

Figure 4 shows the normalized wavelength of Euler and $\mathrm{KdV}$ internal solitary waves of different wave amplitudes as a function of depth for the same stratification discussed above. The wavelength is defined as follows:

$$
\lambda(z)=\frac{1}{2 a(z)} \int_{-\infty}^{\infty} \zeta(x, z) d x .
$$

Here $\zeta(x, z)$ represents the displacement of the isopycnic whose undisturbed depth is $-z$, and $a(z)$ its value at the wave center. Note that $\lambda(z)$ is the equivalent square wavelength (see, e.g., Miyata 1988). In Fig. 4, a normalization was carried out by using the wavelength $\lambda_{H}$ defined at the depth $z=-H_{N}$. Choosing this normalization, the resulting wavelengths of the $\mathrm{KdV}$ internal solitary waves, which do not depend on depth, are also independent of wave amplitude. For every Euler internal solitary wave amplitude, the wavelength increases with increasing depth, this dependence being stronger for larger amplitudes.

We will now discuss the dependence of different characteristics of the simulated large-amplitude internal solitary waves on density stratification. Figure 5 shows the horizontal velocity field of two large-amplitude internal solitary waves as obtained by using the numerical Euler model for the density stratification identified by the first and third set of parameters given in Table 1 . In the case 

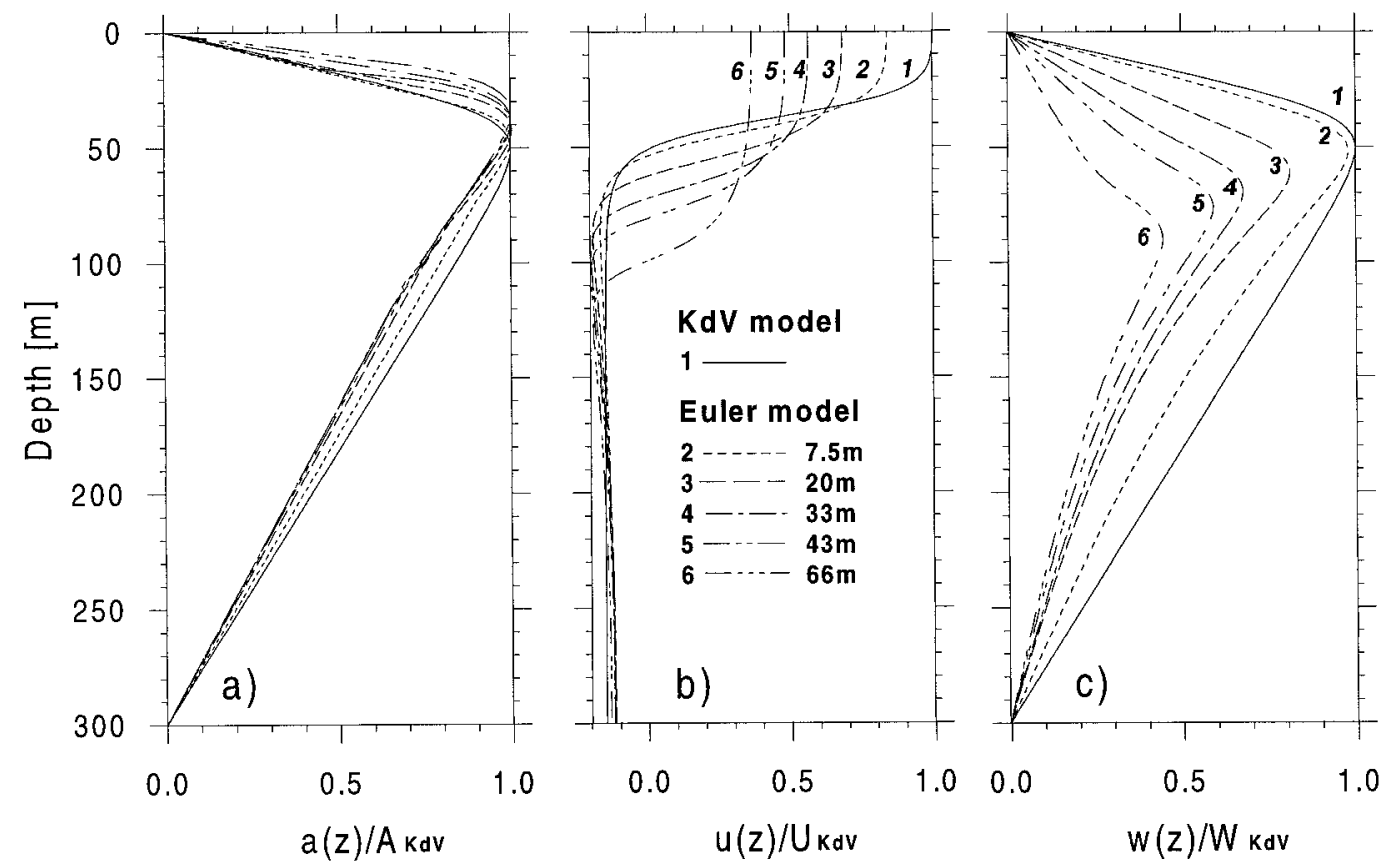

FIG. 3. Vertical profiles of (a) normalized isopycnal displacements calculated at the wave center, (b) normalized horizontal velocities calculated at the wave center, and (c) normalized vertical velocities calculated at the position of maximum vertical velocity as simulated by our Euler model and as calculated by the KdV model for stratification 2 (see Table 1) for different wave amplitudes. The normalization used is explained in the text.

of a density stratification characterized by the presence of a sharp pycnocline (Fig. 5a), that is, for a density stratification close to a two-layer one, a near-surface layer exists where the horizontal velocity is almost independent of depth. Near the pycnocline, however, a

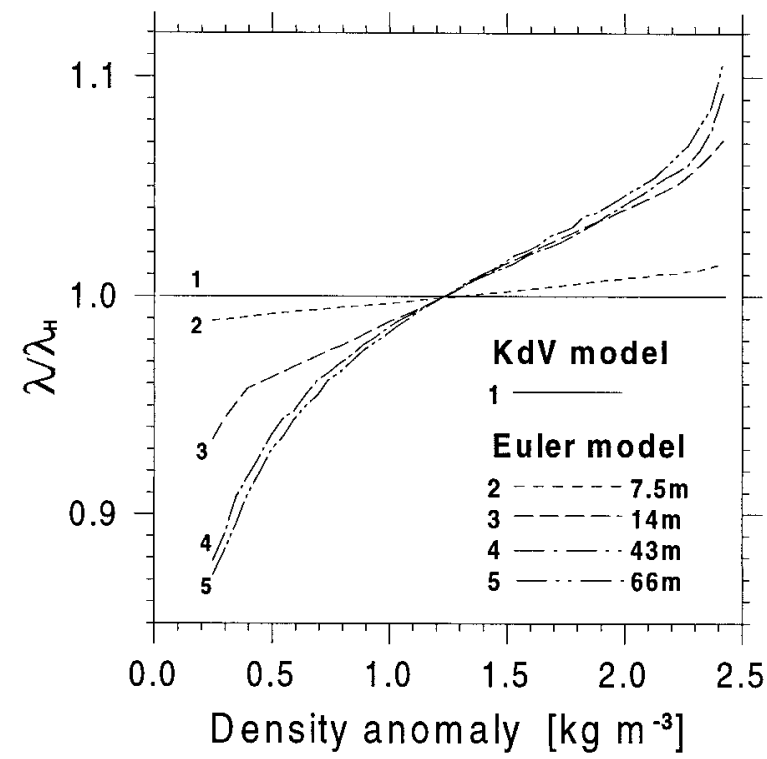

FIG. 4. Normalized wavelengths as a function of the density anomaly as obtained by using our Euler model and as calculated by using the KdV model for stratification 2 (see Table 1) for different wave amplitudes. The normalization used is explained in the text. narrow region characterized by sharp vertical gradients of the horizontal velocity is found where the horizontal velocity reverses. Note that, close to the wave center, the locus of zero horizontal velocity follows the pycnocline, as it would result for an internal wave in a twolayer ocean. On the contrary, in the case of a density stratification characterized by the presence of weak vertical density gradients (Fig. 5b) this locus is an almost horizontal straight line.

In Fig. 6 the wavelength-amplitude relationship (Fig. 6a) as well as the phase speed-amplitude relationship. (Fig. 6b) are plotted for different theoretical models and different density stratifications. The theoretical models include our Euler model, the KdV model, and the Miyata model (Miyata 1988). The results referring to our Euler model and to the $\mathrm{KdV}$ model were calculated for the three density stratifications listed in Table 1, those referring to the Miyata model were calculated for a twolayer stratification in which the depth of the maximum Brunt-Väisälä frequency as well as the surface and bottom densities coincide with the corresponding values common to the three density stratifications listed in Table 1. As discussed above, the Euler model predicts depth-dependent wavelengths. The results of Fig. 6a obtained by using the Euler model refer to the isopycnic whose undisturbed depth coincides with the depth of the maximum Brunt-Väisäla frequency. The bars give the wavelength variation within an internal solitary wave in the density anomaly interval ranging from 0.4 to $2.3 \mathrm{~kg} \mathrm{~m}^{-3}$. For small wave amplitudes the difference 


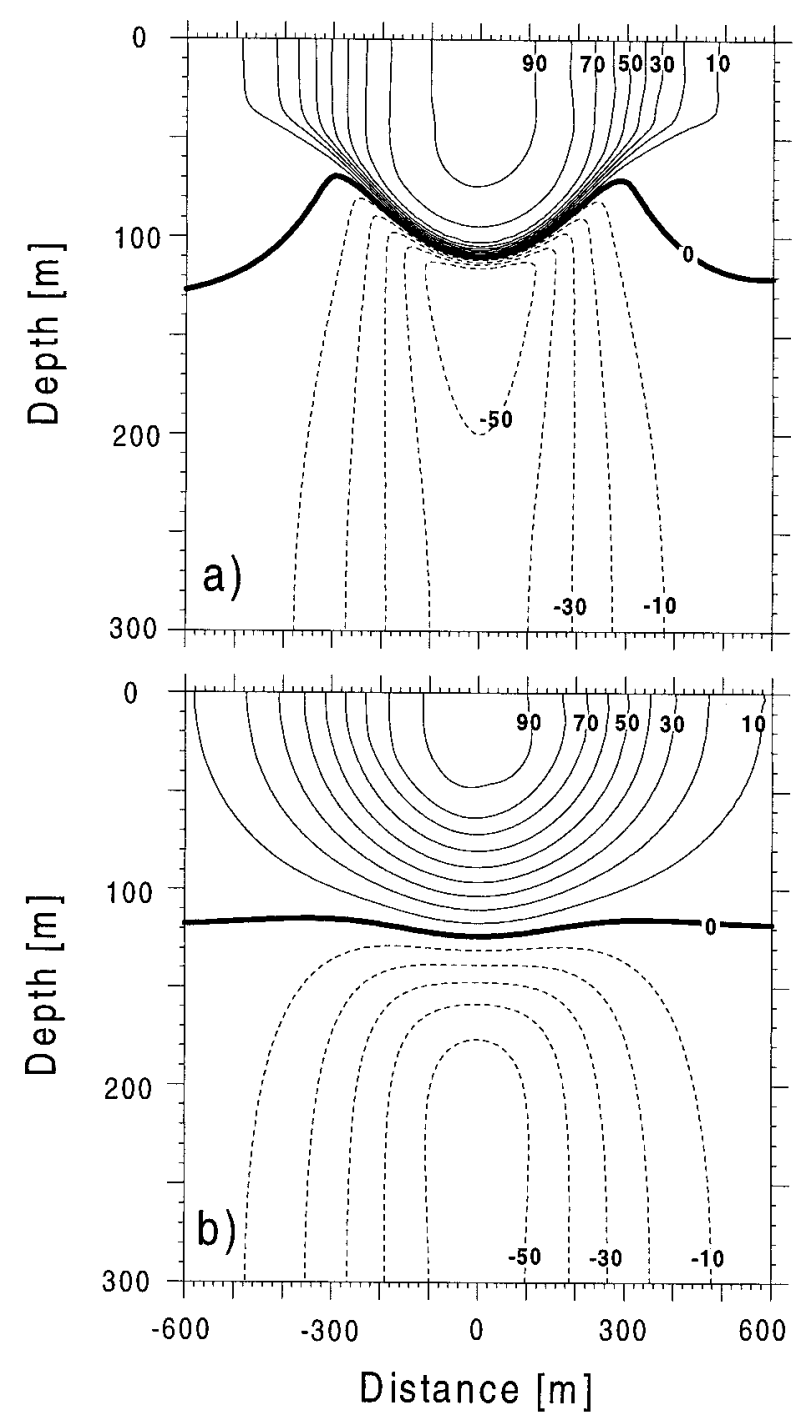

FIG. 5. Fields of horizontal velocity $\left(\mathrm{cm} \mathrm{s}^{-1}\right)$ of large-amplitude internal solitary waves obtained by using our Euler model for (a) stratification 1 and (b) stratification 3 listed in Table 1. Solid lines denote positive horizontal velocities and dotted lines negative horizontal velocities.

in wavelength between Euler and $\mathrm{KdV}$ internal solitary waves is small. This difference increases with increasing amplitude. In contrast to the results of the KdV model, for large amplitudes the wavelength of Euler internal solitary waves increases with increasing amplitude. This characteristic corresponds to a known characteristic of the Miyata model as well as of previous stationary Euler models (Brown and Christie 1998). The dependence of the wavelength of Euler internal solitary waves on depth, given by the bars in Fig. 6a, increases with increasing wave amplitude and decreasing strength of the pycnocline. While in the presence of weak vertical density gradients the difference in phase speed between Euler and KdV internal solitary waves is small, even for large wave amplitudes; for a sharp pycnocline it is
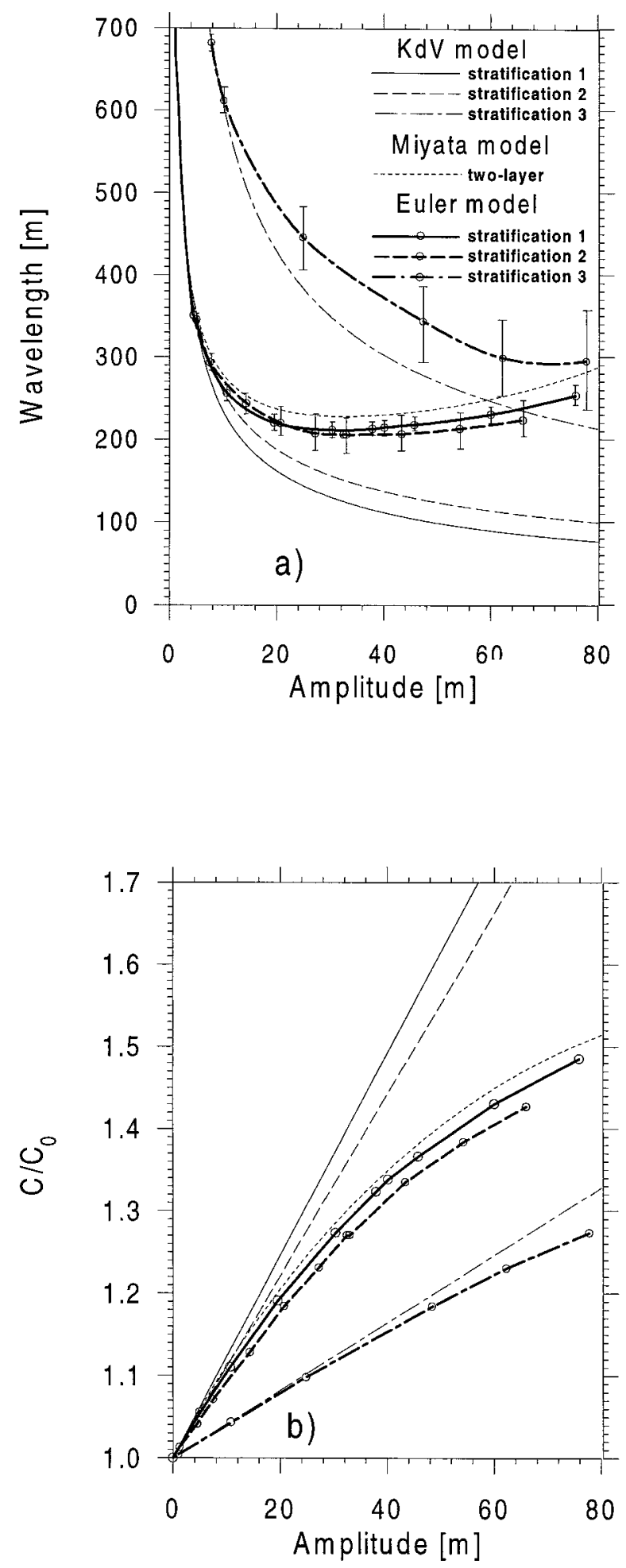

FIG. 6. Relationship between (a) wavelength and wave amplitude and (b) nondimensional phase speed and wave amplitude of internal solitary waves as simulated by our Euler model and as calculated by the KdV model for the stratifications 1-3 (see Table 1), and as calculated by the Miyata model for the two-layer stratification discussed in the text. The nonlinear phase speed $c$ is normalized with the linear phase speed $c_{0}$. 
small only for small wave amplitudes. For these stratifications a much better agreement can be observed between Euler and Miyata internal solitary waves.

\section{b. Observational evidence}

Several of the characteristics of large-amplitude internal solitary waves discussed in the previous section can be observed in the real ocean. In this section we compare high-resolution hydrographic and current data with results obtained by applying our Euler model as well as the KdV model. The hydrographic and current data were acquired north and south of the Strait of Messina, in the central Mediterranean Sea, during the Atlantic Ionian Stream 1995 cruise (AIS'95) and during the Rapid Response 1997 cruise (RR'97) from aboard the NRV Alliance of the SACLANT Undersea Research Centre, La Spezia, Italy. The hydrographic data were obtained using a conventional CTD (conductivity, temperature, depth) probe and a towed CTD chain (Sellschopp 1997); the current data were obtained using a $75 \mathrm{kHz}$ ADCP (acoustic Doppler current profiler). mounted on the vessel. The observations refer to two large-amplitude internal solitary waves measured about $25 \mathrm{~km}$ north and $45 \mathrm{~km}$ south of the strait sill. At these locations a water depth of about $300 \mathrm{~m}$ and $1000 \mathrm{~m}$ respectively was encountered. The observed waves resulted from the disintegration of internal bores generated by the interaction of the barotropic semidiurnal tidal flow with the strait sill (Brandt et al. 1997). Due to the large distance traveled by the waves compared to the typical length scale of the front of the parent internal bore, we feel confident that our data refer to almost stationary internal solitary waves.

For carrying out a comparison between observed and simulated internal solitary waves, a coordinate transformation was applied to the data. As the ship crossed each wave several times, the solitary wave propagation speeds could be estimated. Given these propagation speeds, the measured density and velocity fields could be referred, for each wave, to a common point in time. In Fig. 7a the undisturbed density profiles measured by the CTD chain near the wave fronts are shown. The corresponding Brunt-Väisälä frequency profiles together with the smooth profiles used in the numerical simulations are shown in Fig. 7b. Using these profiles and an initial distubance, the Euler model was run until a stationary solitary wave solution was reached. After several model runs initialized using KdV internal solitary waves of different amplitudes, we were able to produce Euler internal solitary waves having almost the same amplitudes as the measured ones. In Fig. 8 the measured and simulated density (Fig. 8a), horizontal velocity (Fig. 8b), and vertical velocity (Fig. 8c) fields referring to the internal solitary waves north (left panels) and south (right panels) of the Strait of Messina are shown. Several characteristics of the observed and simulated internal solitary waves like, for example, shape
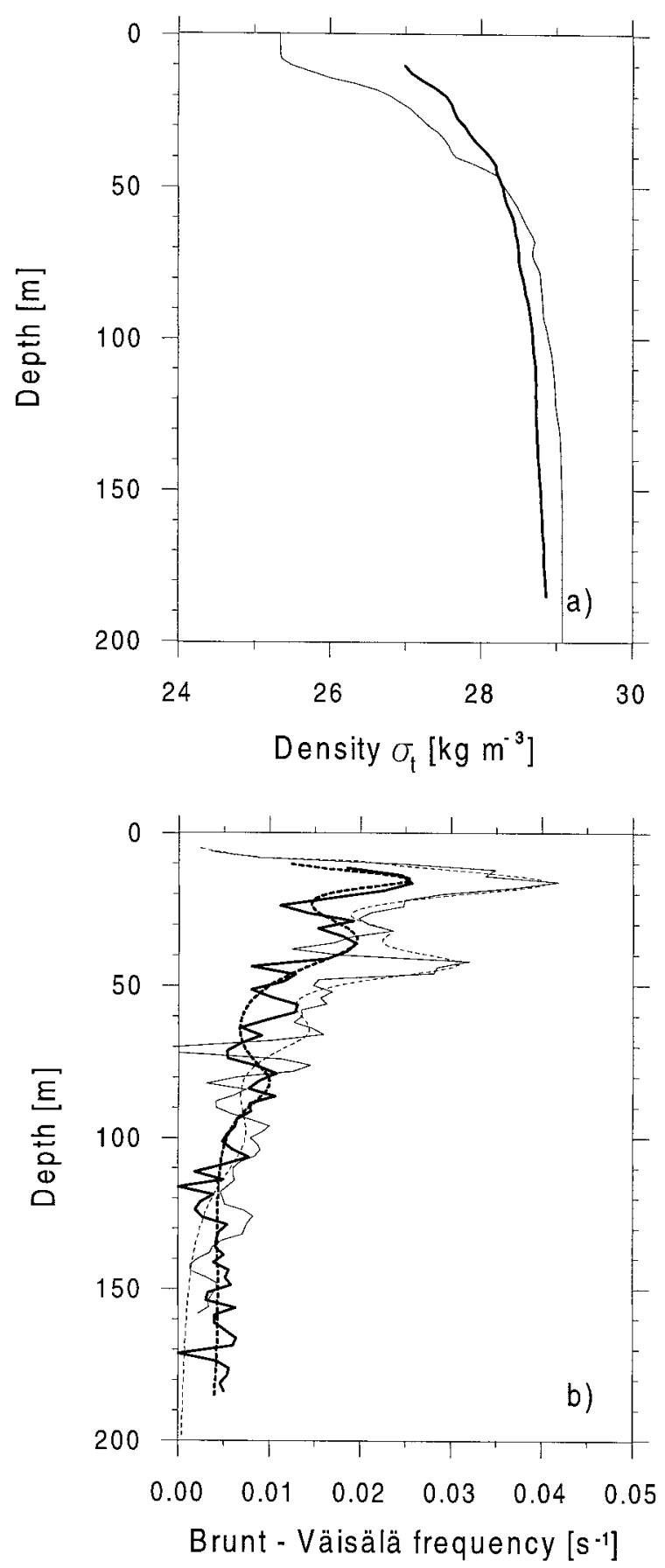

FIG. 7. Undisturbed vertical profiles of (a) densities and (b) BruntVäisälä frequencies measured by the CTD chain near the fronts of the internal solitary waves north (thick lines) and south (thin lines) of the Strait of Messina depicted in Fig. 8. The dotted lines refer to the smooth Brunt-Väisälä frequency profiles used in the numerical simulations.

of the isopycnal displacements, locus of zero horizontal velocity, position of the extrema in the vertical velocity, as well as values of the maximum horizontal and vertical velocity are very similar, thus confirming that our Euler 
a)
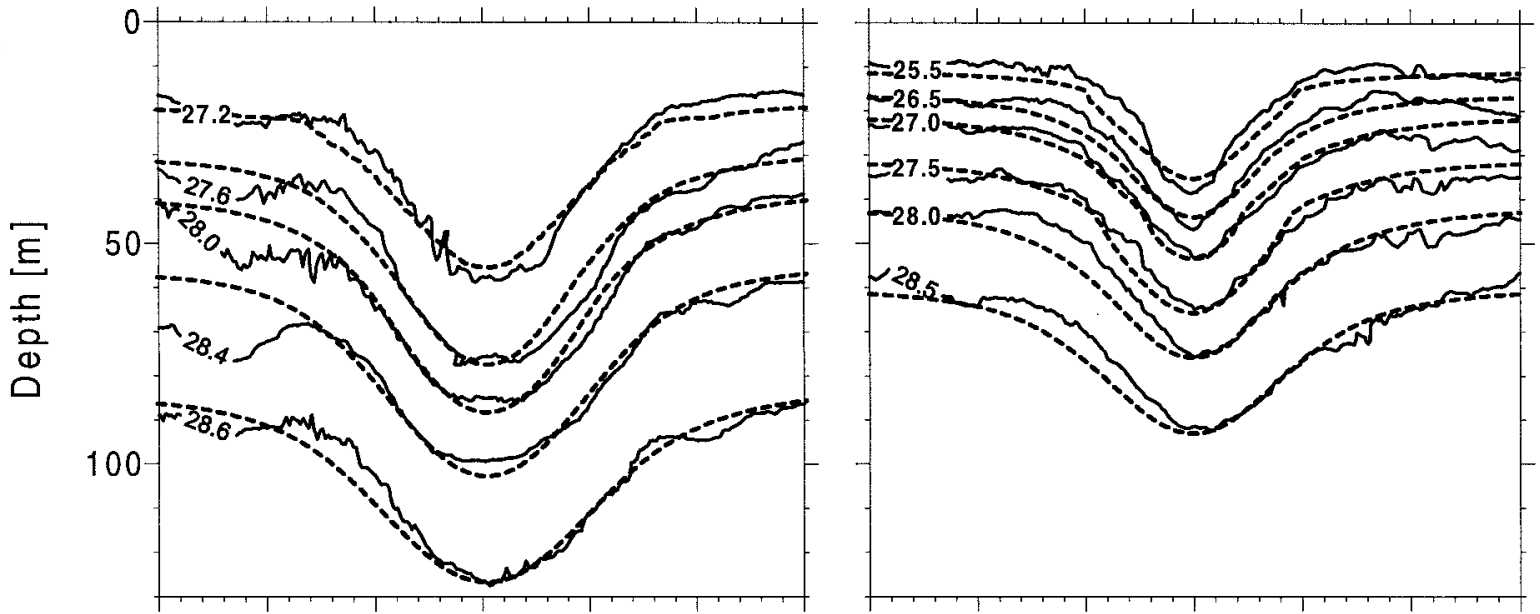

b)
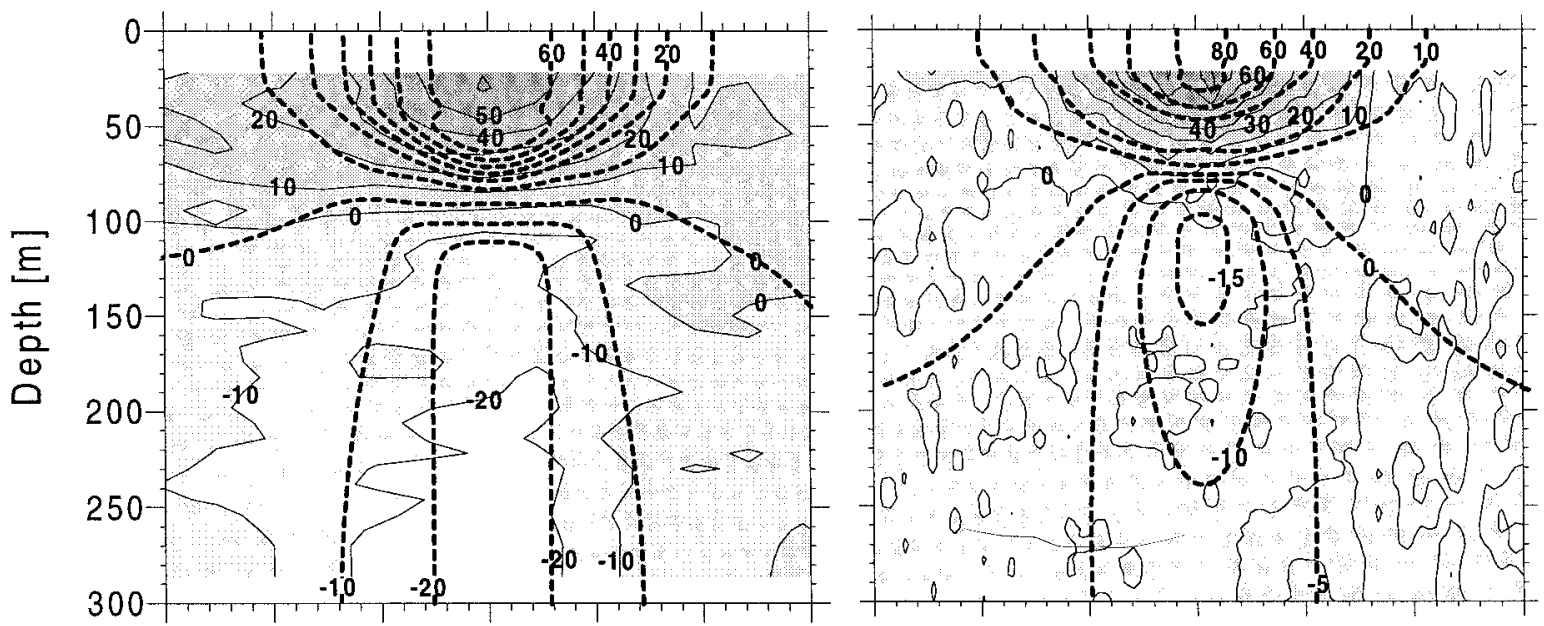

c)

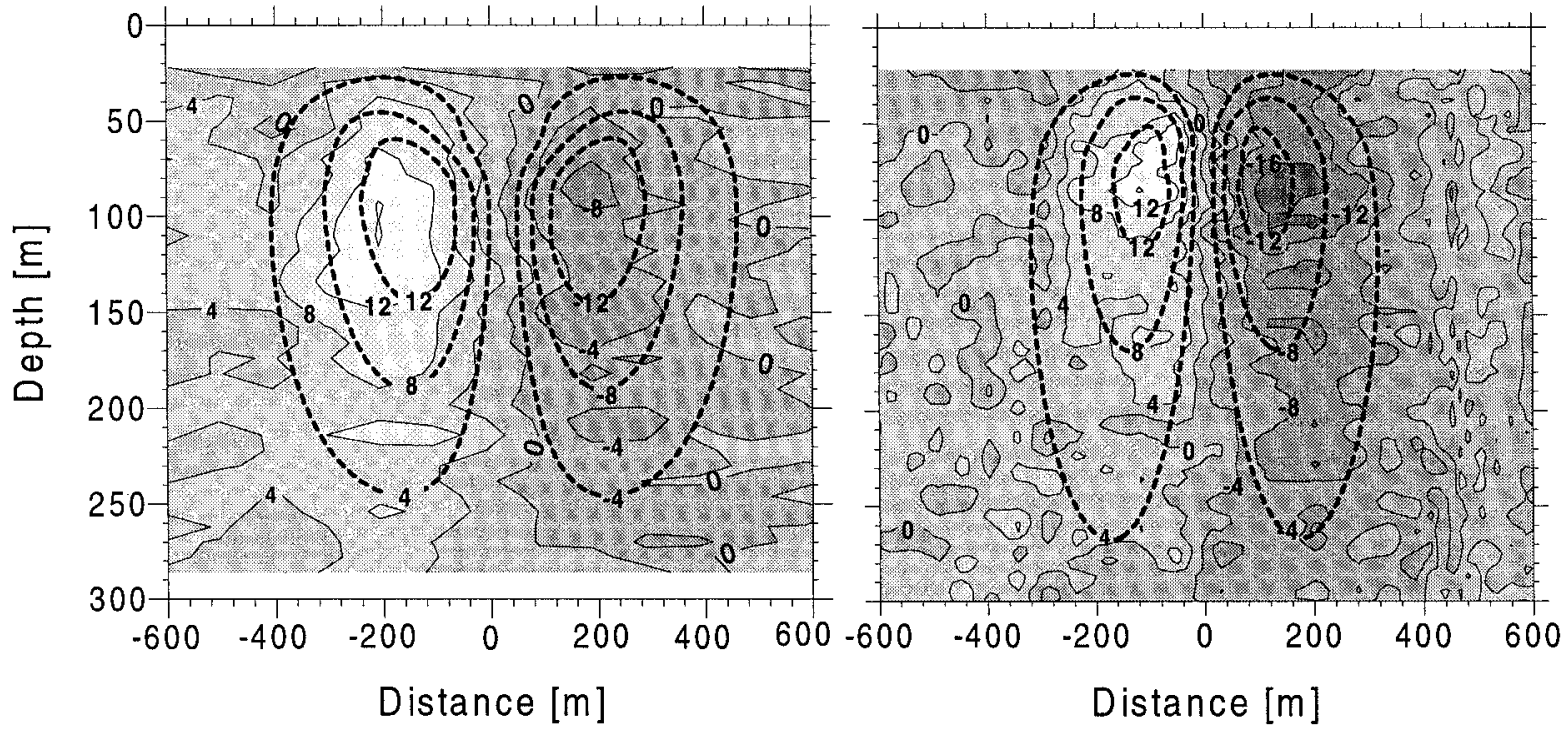

FIG. 8. Fields of (a) density $\left(\mathrm{kg} \mathrm{m}^{-3}\right)$, (b) horizontal velocity $\left(\mathrm{cm} \mathrm{s}^{-1}\right)$, and (c) vertical velocity ( $\left.\mathrm{cm} \mathrm{s}^{-1}\right)$ of two large-amplitude internal solitary waves as measured by the CTD chain and the ADCP north (left panels) and south (right panels) of the Strait of Messina and as simulated by our Euler model (dotted lines). 
TABLE 2. Values of several characteristics of the large-amplitude internal solitary waves north and south of the Strait of Messina as measured by the CTD chain and the ADCP, and as simulated by the Euler model.

\begin{tabular}{|c|c|c|c|c|}
\hline \multirow[b]{2}{*}{ Characteristic } & \multicolumn{2}{|c|}{ North } & \multicolumn{2}{|c|}{ South } \\
\hline & Measured & Simulated & Measured & Simulated \\
\hline Amplitude (m) & 55 & 50 & 34 & 33 \\
\hline Phase speed $\left(\mathrm{m} \mathrm{s}^{-1}\right)$ & 1.0 & 1.0 & 1.2 & 1.1 \\
\hline Max horizontal velocity $\left(\mathrm{m} \mathrm{s}^{-1}\right)$ & 0.61 & 0.67 & 0.81 & 0.83 \\
\hline Max vertical velocity $\left(\mathrm{m} \mathrm{s}^{-1}\right)$ & 0.16 & 0.16 & 0.16 & 0.15 \\
\hline Min vertical velocity $\left(\mathrm{m} \mathrm{s}^{-1}\right)$ & -0.11 & -0.16 & -0.19 & -0.15 \\
\hline $\begin{array}{l}\text { Depth of zero horizontal velocity } \\
\text { at the wave center }(\mathrm{m})\end{array}$ & 94 & 91 & 85 & 77 \\
\hline
\end{tabular}

model is capable of describing the main physical processes governing the dynamics of large-amplitude internal solitary waves in the strait. In Table 2 typical values referring to the observed and simulated internal solitary waves are listed.

A comparison between characteristics of the two internal solitary waves as measured by using the CTD chain and the ADCP, as simulated by using the Euler model, and as calculated by using the $\mathrm{KdV}$ model is presented in Figs. 9 and 10. Figure 9a shows vertical profiles of the isopycnal displacements at the wave center referring to the internal solitary wave north of the Strait of Messina. Note the good agreement between the depth of the maximum isopycnal displacement measured by using the CTD chain and simulated by using our Euler model. In the measured data several local extrema are, however, present that are not captured by our model. They can be a result of the superposition of different subscale internal waves existing in the area. In Fig. 9b the vertical profiles of the horizontal velocity at the wave center referring to the internal solitary wave north of the Strait of Messina are illustrated. A com- parison between the values measured by using the ADCP and simulated by using our Euler model shows a very good agreement in maximum horizontal velocity and depth of zero horizontal velocity. Figure 9c shows vertical profiles of the vertical velocity at the positions of extremal vertical velocity (i.e., at the position where the vertical velocity is maximum, at the rear of the wave, and at the position where it is minimum, at the front of the wave) referring to the internal solitary wave north of the strait. Although the measured data are, in general, noisy, a still good agreement between measured and simulated profiles can be observed. A comparison between the characteristics of the southern internal solitary waves as measured by using the CTD chain and the ADCP and as simulated by using the Euler model reveals also a good agreement (see Fig. 10). As one could expect, from Figs. 9 and 10 it can be clearly evinced that a simple model like the stratified $\mathrm{KdV}$ model is not able to describe several characteristics of the measured internal solitary waves that, on the contrary, can be simulated by using the Euler model. This is particularly evident for the internal solitary wave measured north
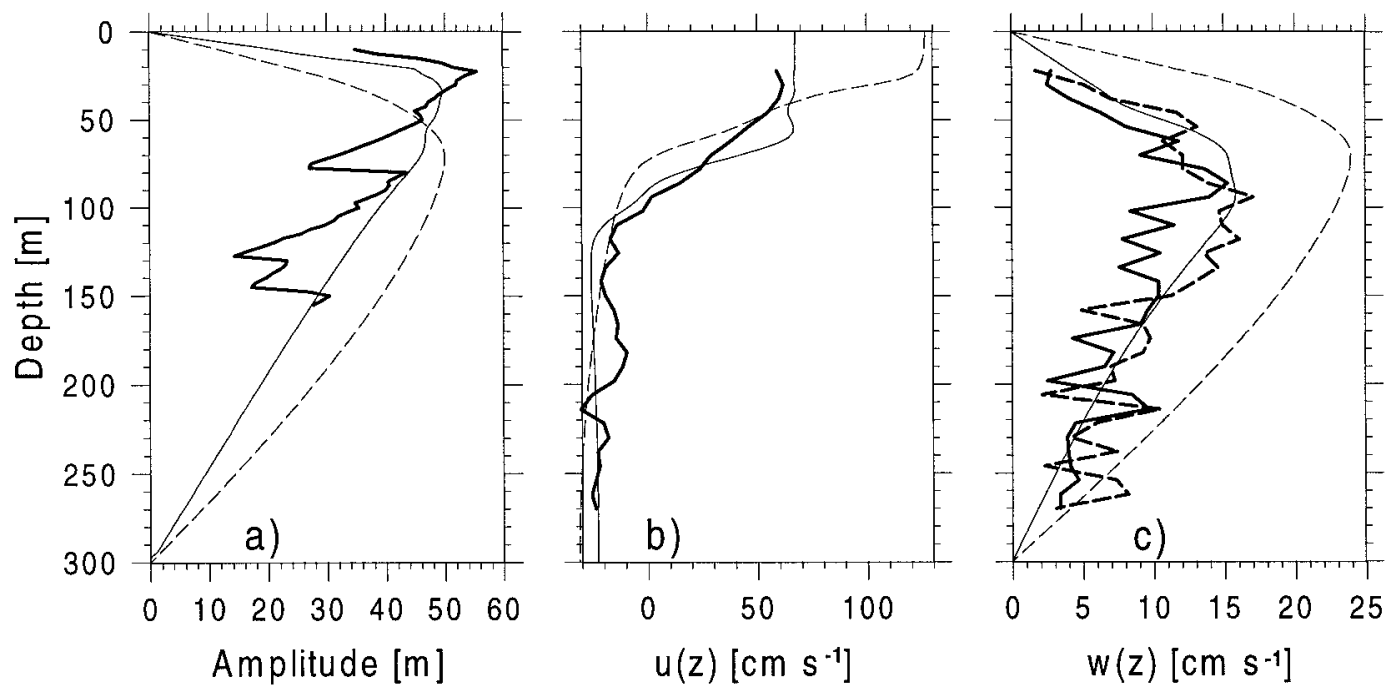

FIG. 9. Vertical profiles of (a) isopycnal displacements calculated at the wave center, (b) horizontal velocities calculated at the wave center, and (c) vertical velocities calculated at the position of extremal vertical velocities as measured by the CTD chain and the ADCP north of the Strait of Messina (thick lines), as simulated by our Euler model (thin solid lines) and as calculated by the KdV model (dashed lines). 

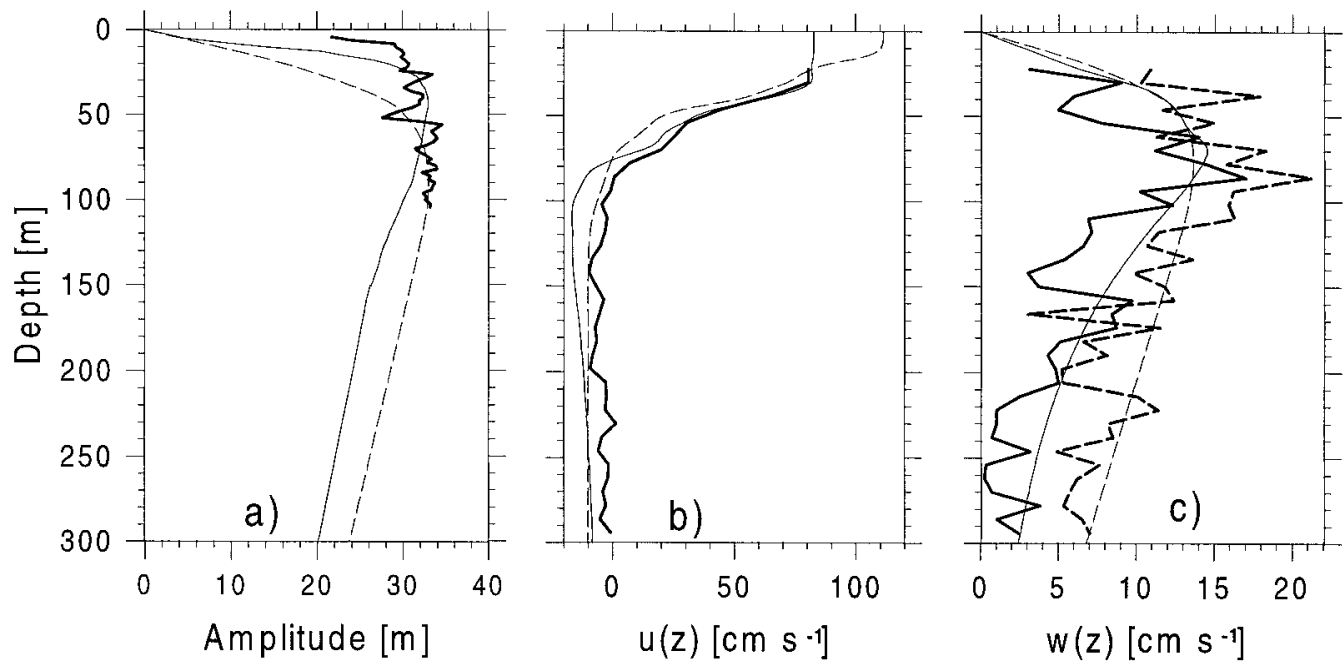

FIG. 10. Vertical profiles of (a) isopycnal displacements calculated at the wave center, (b) horizontal velocities calculated at the wave center, and (c) vertical velocities calculated at the position of extremal vertical velocities as measured by the CTD chain and the ADCP south of the Strait of Messina (thick lines), as simulated by our Euler model (thin solid lines) and as calculated by the KdV model (dashed lines).

of the Strait of Messina, which has a larger amplitude than the one measured south of the strait.

The limit of using the KdV model for the simulation of large-amplitude internal solitary waves becomes particularly evident in the comparison between the wavelength measured by using the CTD chain north and south of the Strait of Messina, simulated by using our Euler model, and calculated by using the KdV model at different depths. This comparison is shown in Fig. 11a for the northern internal solitary wave and in Fig. 11b for the southern one. In fact, while the Euler model is capable of capturing, at least qualitatively, the observed dependence of wavelength on depth, the wavelengths predicted by the KdV model are depth independent.

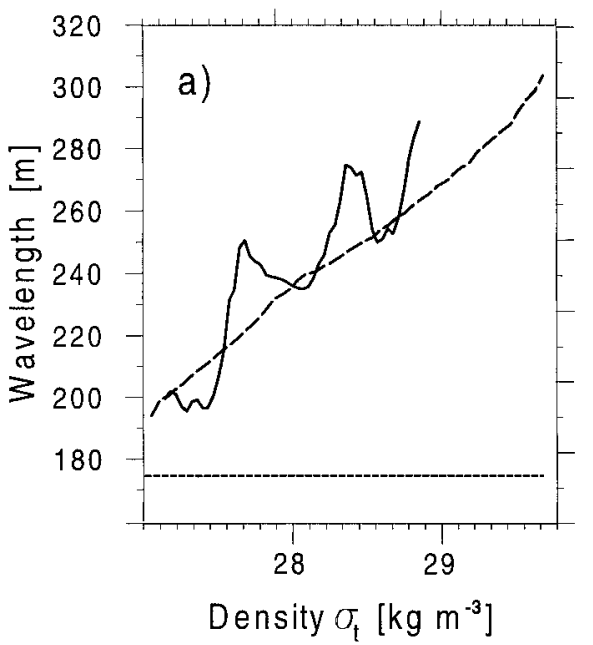

\section{Discussion}

In this paper several aspects of the horizontal and vertical structure of large-amplitude internal solitary waves propagating in stratified waters over a continental shelf as obtained by using an Euler model were discussed. Some of these aspects were found in high-resolution hydrographic and current data referring to internal solitary waves measured north and south of the Strait of Messina. This implies that for a detailed description of the dynamics of large-amplitude internal solitary waves observed in the real ocean higher-order theoretical models like the one used in the present investigation are needed.

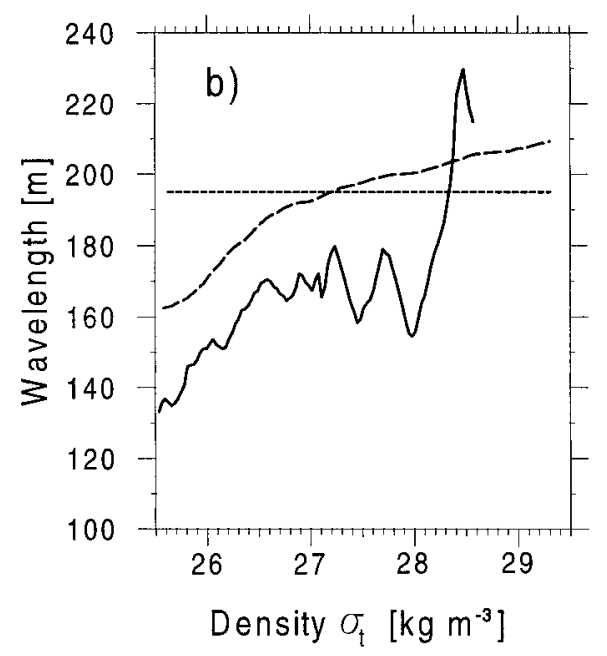

FIG. 11. Wavelength as a function of the density for two large-amplitude internal solitary waves as measured by the CTD chain (a) north and (b) south of the Strait of Messina (solid lines), as simulated by our Euler model (dashed lines), and as calculated by the KdV model (dotted lines). 


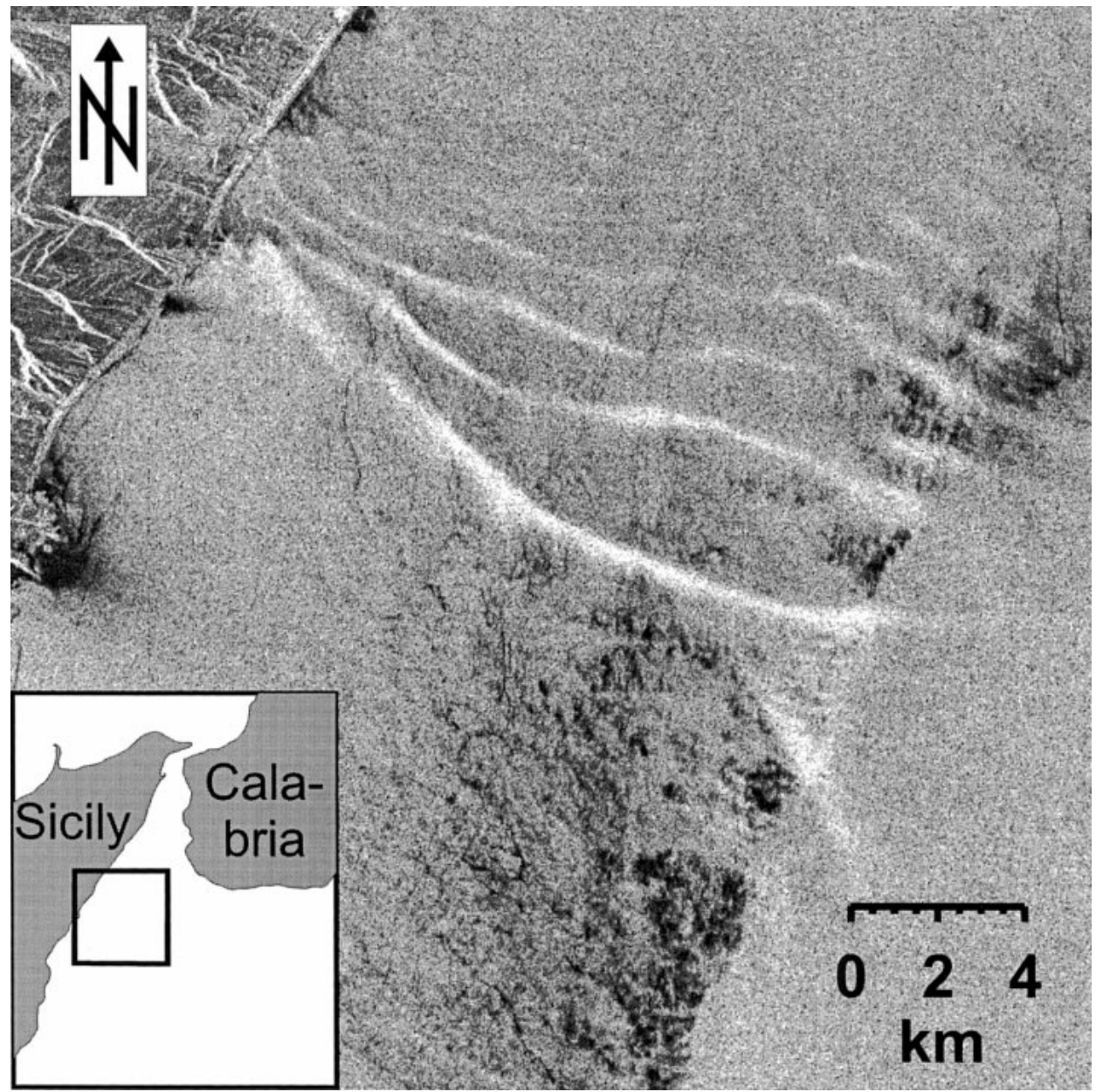

FIG. 12. Radar image of a region south of the Strait of Messina (see inserted geographical map) acquired by the synthetic aperture radar of the European Remote Sensing Satellite-2 at 2113 UTC 22 Aug 1997. The image shows sea surface manifestations of a train of southward propagating internal solitary waves. These sea surface manifestations were observed simultaneously with the in situ measurements discussed in section $3 \mathrm{~b}$. The leading wave pattern is associated to the large-amplitude internal solitary wave whose density and current fields are depicted in Fig. 8, right panels.

Moreover, from the presented results several implications on the complexity connected to the relation between sea surface manifestations of oceanic phenomena as observed by remote sensing and characteristics of the interior ocean emerge. Among the most common features observed by remote sensing in coastal waters are radar signatures of internal solitary waves. They arise from the modulation of the sea surface roughness caused by the variable surface currents associated with internal solitary waves. As an example, a radar image showing sea surface manifestations of a train of southward propagating internal solitary waves south of the Strait of Messina is depicted in Fig. 12. Among the different parameters that can be inferred by the analysis of radar signatures of internal solitary waves are maxima and minima in the backscattered radar signal associated to a single internal solitary wave as well as distance between the locations of these extrema. Note that, in gen- eral, a single solitary wave of depression manifests itself on a radar image as a pattern characterized by the presence of a bright band (corresponding to the wave front) followed by a dark band (corresponding to the wave rear). The difference in brightness between these two bands and the surrounding area can vary greatly depending on wind conditions and radar parameters. In the case of the sea surface manifestations of internal solitary waves visible in Fig. 12, the difference in brightness between the dark band and the surrounding area is not very pronounced. However, the aforementioned parameters represent, in the frame of a first-order radar imaging theory (Alpers 1985), the most promising ones for obtaining characteristics of the wave dynamics and hence information about the interior ocean. A discussion about the complexity of the radar imaging theory of the ocean (and about its possible overcoming, provided that future sensors will be able to measure 

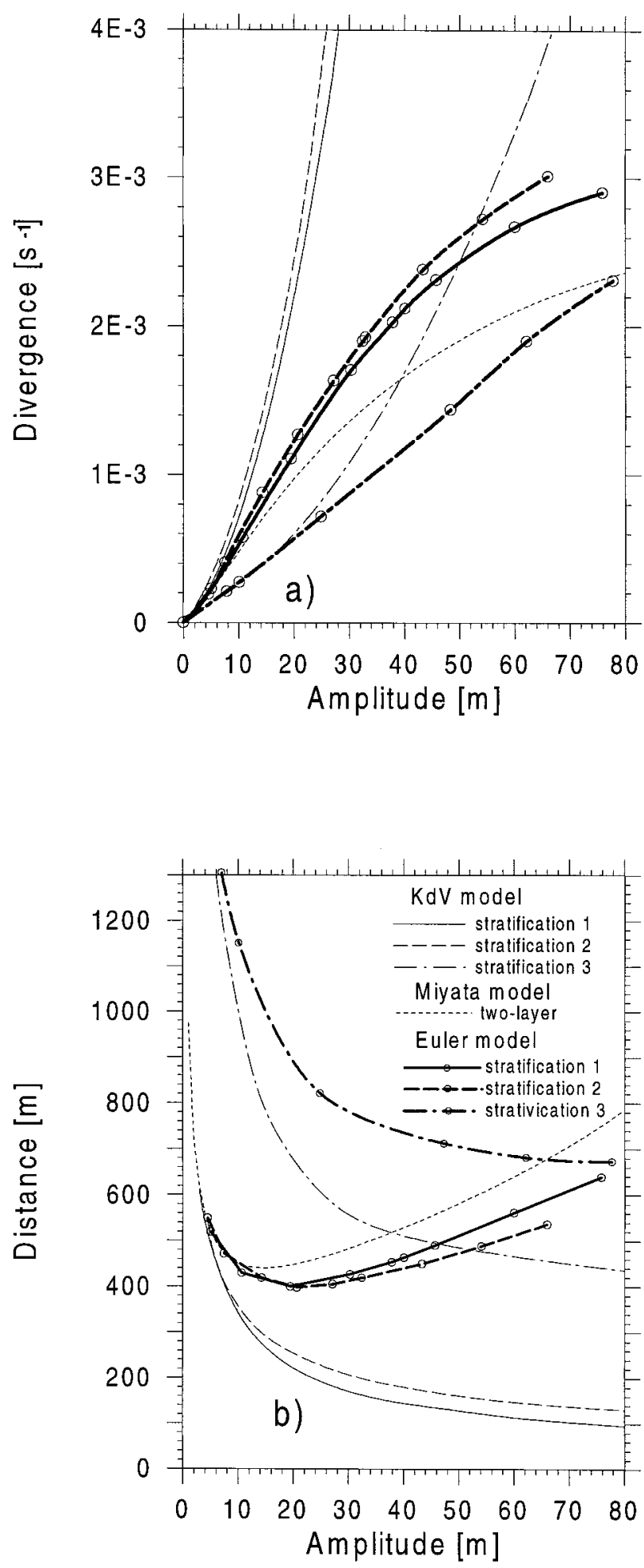

FIG. 13. Relationship between (a) maximum surface velocity divergence and wave amplitude and (b) distance between the locations of maximum surface velocity divergence and convergence and wave amplitude of a single internal solitary wave as simulated by our Euler model and as calculated by the KdV model for the stratifications 1-3 listed in Table 1, and as calculated by the Miyata model for the twolayer stratification discussed in the text. directly parameters of the sea surface velocity field) is beyond the scope of this paper. However, according to the first-order theory mentioned above, the maximum and minimum in the backscattered radar signal associated with a single internal solitary wave, as well as the distance between the locations of these extrema, are related to the maximum surface velocity convergence and divergence and to the distance between the locations of these extrema.

Figure 13 shows the maximum surface velocity divergence (Fig. 13a) and the distance between the locations of maximum surface velocity convergence and divergence of a single internal solitary wave as functions of the wave amplitude (Fig. 13b) as simulated by our Euler model and as calculated by using the $\mathrm{KdV}$ and Miyata models. In the case of the Euler and KdV models, these two parameters are plotted for the three density stratifications listed in Table 1, while, in the case of the Miyata model, for the two-layer stratification discussed in the previous section.

The presented parameters characterizing the sea surface manifestations associated with internal solitary waves depend strongly on the choice of the theoretical models used for their simulation. But they depend also not univocally on the density stratification, a quantity whose temporal and spatial variability is, in general, not known in the World Ocean. This nontrivial dependency can be seen as a measure of the complexity related to the inversion of sea surface manifestations of internal solitary waves into characteristics of the interior ocean.

Acknowledgments. We would like to express our gratitude to Jürgen Sellschopp of the SACLANTCEN, La Spezia, Italy; to Heinz-Volker Fiekas of the "Forschungsanstalt der Bundeswehr für Wasserschall- und Geophysik," Kiel, Germany; and to Detlef Quadfasel of the Institute of Oceanography of the University of Hamburg, Germany. Their efforts made possible the realization of the in situ measurements. We thank Werner Alpers and Nataliya Stashchuk for helpful discussions, Marcus Dengler for processing of the ADCP data, and the captain and crew of the NRV Alliance of the SACLANTCEN for their support during the experimental phase of this study. This work was partly supported by the European Commission under Contract MAS3-CT950027 and by the Deutsche Forschungsgemeinschaft in the frame of the Sonderforschungsbereich 1626.

\section{REFERENCES}

Alpers, W., 1985: Theory of radar imaging of internal waves. Nature, 314, 245-247.

, P. Brandt, A. Rubino, and J. O. Backhaus, 1996: Recent contributions of remote sensing to the study of internal waves in the straits of Gibraltar and Messina. Dynamics of Mediterranean Straits and Channels, F. Briand, Ed., CIESM Science Series No. 2, Bull. Inst. Océanogr. (Monaco), 17, 21-40.

Apel, J. R., H. M. Byrne, J. R. Proni, and R. L. Charnell, 1975: Observations of oceanic internal and surface waves from the 
Earth Resources Technology Satellite. J. Geophys. Res., 80, 865881.

— J. R. Holbrock, A. K. Kiu, and J. J. Tsai, 1985: The Sulu Sea internal soliton experiment. J. Phys. Oceanogr., 15, 1625-1651.

—, L. A. Ostrovsky, and Yu. A. Stepanyants, 1995: Internal solitons in the ocean. Tech. Rep. MERCJRA0695, 70 pp. [Available from Milton S. Eisenhower Research Center, Applied Physics Laboratory, The Johns Hopkins University, Johns Hopkins Rd., Laurel, MD 20707.]

Benney, D. J., 1966: Long non-linear waves in fluid flows. J. Math. Phys., 45, 52-63.

— , and D. R. S. Ko, 1978: The propagation of long large amplitude internal waves. Stud. Appl. Math., 59, 187-199.

Brandt, P., A. Rubino, W. Alpers, and J. O. Backhaus, 1997: Internal waves in the Strait of Messina studied by a numerical model and synthetic aperture radar images from the ERS 1/2 satellites. J. Phys. Oceanogr., 27, 648-663.

—, D. Quadfasel, W. Alpers, J. Sellschopp, and H.-V. Fiekas, 1999: Evidence for the influence of Atlantic-Ionian stream fluctuations on the tidally induced internal dynamics in the Strait of Messina. J. Phys. Oceanogr., 29, 1071-1080.

Brown, D. J., and D. R. Christie, 1998: Fully nonlinear solitary waves in continuously startified incompressible fluids. Phys. Fluid, 10, $2569-2586$.

Davis, R. E., and A. Acrivos, 1967: Solitary internal waves in deep water. J. Fluid Mech., 29, 593-607.

Djordjevic, V. D., and L. G. Redekopp, 1978: The fission and disintegration of internal solitary waves moving over two-dimensional topography. J. Phys. Oceanogr., 8, 1016-1024.

Dubreil-Jacotin, M. K., 1937: Sur les théoremes d'existence relatifs aux ondes permanentes pérodiques à deux dimensions dans les liquides hétérogénes. J. Math. Pure Appl., 16, 43.

Funakoshi, M., and M. Oikawa, 1986: Long internal waves of large amplitude in a two-layer fluid. J. Phys. Soc. Japan, 55, 128 144.

Gear, J. A., and R. A. Grimshaw, 1983: Second-order theory for solitary waves in shallow fluids. Phys. Fluids, 26, 14-29.

Grimshaw, R., E. Pelinovsky, and T. Talipova, 1997: The modified Korteweg-de Vries equation in the theory of large-amplitude internal waves. Nonlin. Proc. Geophys., 4, 237-250.
Konyaev, K. V., K. D. Sabinin, and A. N. Serebryany, 1995: Largeamplitude internal waves at the Mascarene Ridge in the Indian Ocean. Deep-Sea Res., 42, 2075-2091.

Koop, C. G., and G. Butler, 1981: An investigation of internal solitary waves in a two-fluid system. J. Fluid Mech., 112, 225-251.

Lamb, K. G., and L. Yan, 1996: The evolution of internal wave undular bores: Comparison of a fully nonlinear numerical model with weakly nonlinear theory. J. Phys. Oceanogr., 26, 27122734.

Lee, C., and R. C. Beardsley, 1974: The generation of long nonlinear internal waves in a weakly stratified shear flow. J. Geophys. Res., 79, 453-462.

Long, R. R., 1953: Some aspects of the flow of stratified fluids. I. A theoretical investigation. Tellus, 5, 42-58.

Marchuk, G. I., 1974: Numerical Methods in Weather Prediction. Academic Press, 277 pp.

Michallet, H., and E. Barthelemy, 1998: Experimental study of interfacial solitary waves. J. Fluid Mech., 366, 159-177.

Miles, J. W., 1979: On internal solitary waves. Tellus, 31, 456-462. , 1981: On internal solitary waves II. Tellus, 33, 397-401.

Miyata, M., 1988: Long internal waves of large amplitude. Nonlinear Water Waves, K. Horikawa and H. Maruo, Eds., Springer-Verlag, 399-406.

Munk, W., and C. Wunsch, 1998: Abyssal recipes II: Energetics of tidal and wind mixing. Deep-Sea Res., 45, 1977-2010.

Osborne, A. R., and T. I. Burch, 1980: Internal solitons in the Andaman Sea. Science, 208, 451-269.

Ostrovsky, L. A., and Yu. A. Stepanyants, 1989: Do internal solitons exist in the ocean? Rev. Geophys., 27, 293-310.

Sellschopp, J., 1997: A towed CTD chain for high-resolution hydrography. Deep-Sea Res., 44, 147-165.

Terez, D. E., and O. M. Knio, 1998: Numerical simulations of largeamplitude internal solitary waves. J. Fluid Mech., 362, 53-82.

Tung, K. K., T. F. Chan, and T. Kubota, 1982: Large-amplitude internal waves of permanent form. Stud. Appl. Math., 65, 1.

Turkington, B., A. Eydeland, and S. Wang, 1991: A computational method for solitary waves in a continuously stratified fluid. Stud. Appl. Math., 85, 93.

Vlasenko, V. V., 1994: Multi-modal soliton of internal waves. Atmos. Oceanic Phys., 30, 161-169. 